\title{
Amélioration de l'état nutritionnel des enfants âgés de 6 à 30 mois à Lissèzoun (Centre-Bénin) par la poudre de feuilles de Moringa oleifera (Lam.)
}

\author{
Bidossessi Victor Saturnin HOUNDJI ${ }^{1,2^{*}}$, Sam F. BODJRENOU ${ }^{1}$, Serge B. Marius \\ LONDJI ${ }^{1}$, Romaric OUETCHEHOU ${ }^{1}$, Alfred ACAKPO ${ }^{3}$, Kou'Santa Sabiba E. \\ AMOUZOU $^{2}$ et Dr. HOUNMENOU ${ }^{4}$ \\ ${ }^{1}$ Faculté des Sciences Agronomiques, Université d'Abomey-Calavi, 01 BP 526 Cotonou, Bénin. \\ ${ }^{2}$ Faculté des Sciences - Département de Biochimie/Nutrition, Université de Lomé. BP 1515, Lomé. Togo. \\ ${ }^{3}$ Clinique de Recherche en Santé et en Nutrition, BP 1001 Porto-Novo, Bénin. \\ ${ }^{4}$ Service de Pédiatrie, Hôpital BETHESDA, Cotonou, Bénin. \\ *Auteur correspondant ; E-mail: saturninh@yahoo.fr ; Tel: (00 229) 97983697 ; 05 BP 1225 Cotonou, \\ République du Bénin.
}

\section{RESUME}

Le but de la présente étude est d'apprécier l'effet d'une prise quotidienne de $10 \mathrm{~g}$ de poudre de feuilles de Moringa oleifera (PFMo) sur l'état nutritionnel des enfants de 6 à 30 mois d'âge souffrant de malnutrition aigüe modérée après 6 mois de supplémentation. Deux groupes ont été constitués à partir de 84 nourrissons choisis dans un centre de récupération nutritionnelle du village de Lissèzoun (Centre-Bénin) : l'un a reçu la PFMo et l'autre, choisi comme témoin n'a pas reçu cette poudre. L'état nutritionnel des enfants a été apprécié par des indicateurs anthropométriques. A la fin des 6 mois, les résultats montrent que la supplémentation quotidienne en PFMo a amélioré significativement l'état nutritionnel des enfants aussi bien pour l'émaciation (Z-score Poids/Taille de $-1,0 \pm 0,9$ au début de l'intervention à $0,7 \pm 1,0$ à la fin des 6 mois), le retard de croissance (Z-score Taille/Age de $-2,6 \pm 0,7$ à $0,4 \pm 0,7$ ) que pour l'insuffisance pondérale (Z-score Poids/Age de $-2,2 \pm 0,6$ à $0,7 \pm 0,7$ ). L'amélioration des Z-scores a été plus importante dans le groupe d'intervention que dans le groupe témoin et s'est traduite par des prévalences nulles pour les trois types de malnutrition à la fin de la période d'expérimentation.

(C) 2013 International Formulae Group. All rights reserved.

Mots clés: Poudre de feuilles de Moringa oleifera, malnutrition aigue modérée, supplémentation, anthropométrie.

\section{INTRODUCTION}

Les malnutritions sont des problèmes de santé publique. Depuis 10 ans, la sousalimentation connaît une augmentation lente mais constante dans le monde. Entre 2008 et 2009, le nombre de personnes sous-alimentées dans le monde a augmenté de $10 \%$ dont plus $\mathrm{du}$ quart pour l'Afrique au Sud du Sahara (FAO, 2009). Les enfants constituent la couche la plus vulnérable. La malnutrition contribue à $35 \%$ des décès d'enfants de moins de cinq ans en Afrique de l'Ouest et du Centre. Actuellement, un million d'enfants de cette tranche d'âge meurent chaque année dans cette région des causes associées à la malnutrition (UNICEF, 2010). Les formes graves de malnutrition comprennent entre autres le marasme et le kwashiorkor. 
Les pays en développement, à l'instar du Bénin, connaissent des taux de prévalence élevés de malnutrition. Ainsi, selon les dernières données disponibles au plan national (FAO, 2011) en 2008, près de $40 \%$ des enfants âgés de 6 à 59 mois étaient atteints de retard de croissance, un niveau de prévalence qui reste très élevé malgré une tendance à la baisse depuis le début des années 2000. La prévalence de maigreur est de $5 \%$. Au nombre des principaux déterminants de la prévalence élevée de malnutrition chronique, nous pouvons citer notamment la persistance de pratiques d'alimentation inadéquates des jeunes enfants et l'alimentation de complément peu diversifiée. Et pourtant, les populations disposent de ressources alimentaires forestières insoupçonnées parmi lesquelles les légumes feuilles. Les produits forestiers en général et les légumes feuilles en particulier contribueraient à la résolution d'un tel problème au niveau des populations africaines. Plusieurs espèces de légumes feuilles traditionnels d'Afrique sont riches en micronutriments (Schippers, 2002 ; AbukutsaOnyango, 2007). Ils sont d'importantes sources de vitamines (carotène, acide ascorbique, et riboflavine), des protéines et des minéraux tels que le fer et le calcium (Tchiegang et Kitikil, 2004; Akubugwo et al., 2007; Avallone et al., 2007; Ndong et al., 2007; Afoloyan et Jimoh, 2009).

En plus de leurs qualités alimentaires et nutritionnelles, les légumes feuilles ont des propriétés curatives, régulatrices et/ou stimulatrices (Dansi et al., 2008). Certains légumes feuilles sont considérés comme de véritables plantes médicinales. Ils sont donc, ou peuvent être, utilisés comme médicaments (Dansi et al., 2008). Ainsi, beaucoup de légumes feuilles sont utilisés dans le traitement de nombreuses affections. C'est le cas du Moringa oleifera (Lam.) dont les extraits foliaires présentant une activité antimicrobienne importante et permettraient d'améliorer la réponse immunitaire de l'organisme (Yang et al., 2006). Au Bénin et plus particulièrement dans la partie Sud du pays, le $M$. oleifera est régulièrement consommé par les femmes enceintes pour prévenir l'anémie (Dansi et al., 2008). Les légumes feuilles apparaissent donc aujourd'hui comme un moyen de lutte contre la «faim cachée», c'est-à-dire les carences en micronutriments comme la vitamine $\mathrm{A}$ et les minéraux dont le fer (Dansi et al., 2008). Moringa oleifera (Lam.) est de la famille des Moringaceae, qui fait partie de l'ordre des Capparacées. La famille des Moringaceae ne comporte qu'un seul genre, Moringa, qui regroupe 13 espèces ; c'est un arbre pérenne, à croissance rapide qui peut atteindre 7 à 12 mètres de hauteur. Cette plante, localement désignée en Fon et Goun (langues de la République du Bénin) sous le nom de «Kpatinman» fait l'objet de plusieurs usages thérapeutiques au Bénin: traitement des courbatures, de la fièvre, des céphalées, des convulsions, des coliques, de la perte d'appétit et des soins des bébés chétifs (Dansi et al., 2008). Récemment, certaines études en nutrition ont été initiées sur des plantes en vue d'en extraire les protéines foliaires. Les résultats de ces dernières ont permis l'utilisation de ces plantes pour améliorer le statut nutritionnel des enfants qui développent certaines carences alimentaires et nutritionnelles. Des études complémentaires sur l'utilisation de ces extraits ont été conduites aux plans hygiénique et sanitaire dans le but de leur utilisation rationnelle (Guyot et al., 2010). Certains auteurs ont rapporté que la poudre obtenue à partir des feuilles sèches de $M$. oleifera a une concentration en nutriments plus élevée que celle des feuilles fraîches. Elle est donc utilisée de plus en plus comme complément alimentaire par les populations rurales. Le but de ce travail qui est une première au Bénin, est d'apprécier l'aptitude de la PFMo, à améliorer l'état nutritionnel parmi les enfants âgés de 6 à 30 mois.

\section{MATERIEL ET MÉTHODES}

\section{Matériel végétal: poudre de feuilles de Moringa}

Les feuilles de $M$. oleifera utilisées pour la production de la poudre proviennent des champs des producteurs et transformateurs de l'Association des Promoteurs et Producteurs des Essences Forestières (APPEF) du village Tchèkpo-Dédékpoé 
(Tabligbo) au Sud du Togo, pour des raisons de disponibilité et d'accessibilité. Les feuilles ont été prélevées entre $09 \mathrm{~h}$ et $10 \mathrm{~h}$ GMT. Cette précaution permet d'éviter le prélèvement de feuilles humidifiées par la rosée. Ces feuilles sont prélevées sur des plants âgés d'au moins six mois.

Le diagramme de production de la poudre de feuilles de Moringa oleifera (PFMo) suit trois grandes étapes constituées d'opérations unitaires simples de salubrité des feuilles de Moringa. Les feuilles sont triées, lavées et essuyées. Elles sont ensuite séchées à l'ombre dans une pièce bien aérée et naturellement ventilée. Après séchage, les feuilles sont broyées et tamisées. La poudre ainsi obtenue est immédiatement conditionnée dans différents emballages tels que des seaux plastiques opaques de $15 \mathrm{~kg}$, des bocaux de $500 \mathrm{~g}, 250 \mathrm{~g}$ et $125 \mathrm{~g}$ et laissée à la température ambiante (Figure 1). La PFMo ainsi produite ne subit aucun autre traitement spécial et est exempte d'additifs alimentaires. Ce mode de production des protéines foliaires avait été décrit précédemment par Amorigi (1980) et Houinsou (1990) dans les opérations de séchage des fruits et légumes.

\section{Composition de la PFMo}

Le Tableau 1 issu de la compilation de plusieurs valeurs moyennes, présente la composition de la PFMo en certains nutriments et le taux de satisfaction des besoins journaliers pour un enfant de 1 à 3 ans.

\section{Population d'étude}

Quatre-vingt-huit enfants diagnostiqués malnutris aigus modérés $(-3<$ Z-score Poids/taille $<-2$, normes OMS) ont été recrutés dans un centre de récupération nutritionnelle d'une Organisation Non Gouvernementale : ONG-Enagnon Dandan de l'arrondissement de Lissèzoun, commune de Bohicon (département du Zou/Collines). Bohicon est situé à $140 \mathrm{~km}$ de Cotonou, la capitale économique du Bénin. Les enfants ont été ensuite répartis en deux groupes : le groupe "Etude" sous PFMo (46 enfants) comme complément alimentaire et le groupe "Témoin" (42 enfants) qui ne reçoit pas la
PFMo. Les mères ou tutrices (celles qui ont la garde des enfants) reçoivent par quinzaine pour leur enfant et ceci pendant 6 mois un stock de petits sachets de médicament générique contenant chacun $10 \mathrm{~g}$ de PFMo. Chaque petit sachet correspond à la dose journalière de PFMo. La poudre est mélangée à l'aliment prêt à être consommé, en une ou deux prises, entre $8 \mathrm{~h}$ et $9 \mathrm{~h}$ le matin et entre $17 \mathrm{~h}$ et $18 \mathrm{~h}$ dans l'après-midi. Afin de protéger la PFMo de la lumière et de l'humidité, les petits sachets sont emballés dans un double sachet en plastique noir.

L'âge des enfants a été estimé à partir de leur date de naissance telle que figurée sur l'acte de naissance, la fiche de naissance ou le carnet de santé. Il en est de même des données relatives au statut vaccinal. Les statuts socio-économiques des parents ont été collectés grâce à un questionnaire. Pour l'enrôlement des enfants, des critères ont été fixés. Critère d'inclusion: au moment du recrutement les enfants devraient être âgés de 6 mois à 30 mois \pm 7 jours, être allaités maternellement par leur mère ou leur tutrice, répertoriés dans la base de données du Centre de Récupération Nutritionnelle, diagnostiqués malnutris modérés et appartenir à une famille ne prévoyant pas un déplacement ou un voyage de plus d'une semaine. Critère d'exclusion : tout enfant porteur de maladies chroniques comme les insuffisances rénales, le diabète, les broncho-pneumopathies, les hémoglobinopathies homozygotes et les enfants atteints d'autres handicaps sévères ont été exclus de l'échantillon.

Les mères/tutrices ont été sensibilisées auparavant lors de différentes séances de pesées sur les objectifs de l'étude. Elles ont, après concertation avec le chef de ménage, accepté de participer librement à cette étude en signant un engagement de consentement. Des enquêteurs locaux ont été recrutés pour le suivi en ambulatoire de la prise régulière et effective de la PFMo par les enfants ciblés. Le suivi se fait trois fois par semaine dont une fois en visite inopinée afin de s'assurer de l'effectivité de la prise de la PFMo. Afin que l'étude ne soit pas perçue comme une intervention discriminante, les mères des 


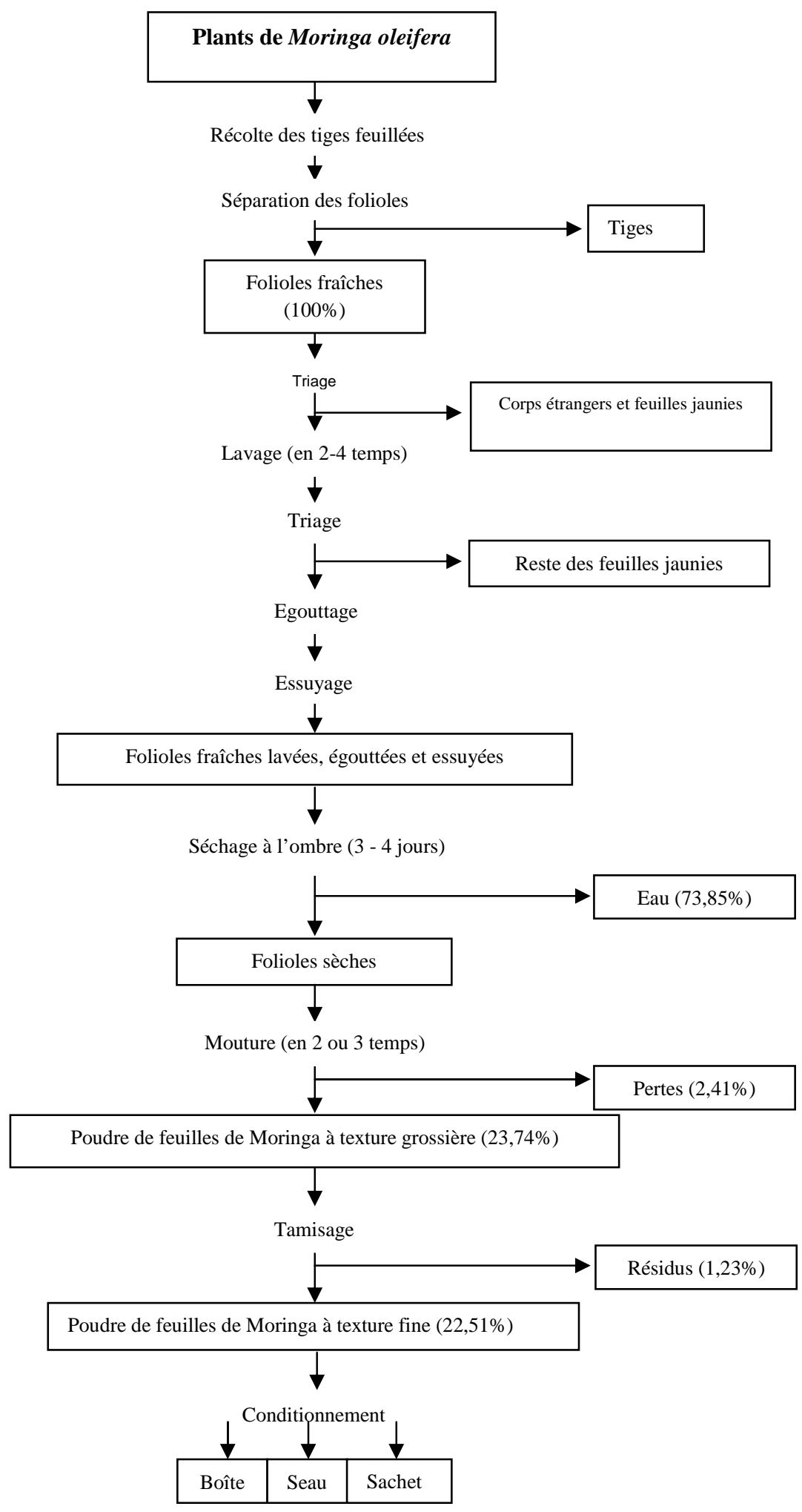

Figure 1 : Diagramme technologique de production de la poudre de feuilles de Moringa. 
enfants du groupe "Témoin" ont reçu également à la fin de l'intervention de la PFMo pour leur enfant.

\section{Instruments et outils de collecte des données}

L'unité de recherche est constituée par le couple mère/enfant. Les mères ou tutrices ont été associées à ces différentes opérations. Les mesures anthropométriques ont été prises au siège du bureau de l'arrondissement de Lissèzoun.

En fonction de l'âge et de la capacité à se tenir debout des enfants, la taille a été mesurée en position couchée ou allongée (taille couchée ou longueur) ou en position debout (taille debout). Deux instruments ont été alors utilisés: une toise coulissante et graduée (Infantomètre) déposée sur une table à un emplacement plan pour les enfants âgés de 6 mois à 18 mois et une micro-toise pour les plus âgés. Ainsi, pour les enfants de moins de $85 \mathrm{~cm}$ (enfants de moins de deux ans), ou ceux, trop faibles pour se tenir debout, on mesure la "longueur" de l'enfant en supination c'est-à-dire en position allongée. Pour les enfants de $85 \mathrm{~cm}$ ou plus (enfants de deux ans ou plus), on mesure la taille en position debout avec la micro-toise appelée encore stadiomètre ou toise horizontale. Chaque enfant a été mesuré trois fois. La valeur retenue est la moyenne des trois mesures. Pour la prise du poids, une balance électronique à piles, de type Seca 354 «lena » de portée maximale de $20 \mathrm{Kg}$, de graduation $0,01 \mathrm{Kg}<10 \mathrm{Kg}>0,02 \mathrm{~g}$ et de précision \pm 30 $\mathrm{g}$ ou $\pm 0,6 \%$ a été utilisée. Elle est déposée sur une surface stable et plane. Ce type de balance permet de peser les bébés en position couchée et en position debout selon la commodité des enfants. Chaque enfant a été pesé trois fois. La valeur retenue est la moyenne des trois pesées.

\section{Logiciels utilisés}

Pour caractériser et apprécier l'état nutritionnel des enfants, les indices anthropométriques ont été calculés à l'aide du logiciel Anthro V3.0.1. Ces indices ont été exprimés en écart-type (ET) ou Z-score. Il s'agit du Z-score poids pour taille $(P T Z)<-$ 2ET, cet indice caractérise l'état de maigreur et traduit une forme de malnutrition aiguë ; du Z-score taille pour âge (TAZ) < -2ET, cet indice caractérisant le retard de croissance traduit une forme de malnutrition chronique et du Z-score poids pour âge $(P A Z)<-2 \mathrm{ET}$ qui caractérise l'insuffisance pondérale chez les enfants, forme mixte entre malnutrition aiguë et chronique. Le logiciel MinTab 14.0 a été utilisé pour réaliser les tests statistiques de comparaison de moyennes (tests $t$ de Student) et le Tableur Excel de Microsoft Office 2007 pour l'estimation des statistiques descriptives (moyennes et écart-types). Les conclusions ont été tirées à un seuil de probabilité de $5 \%$.

\section{RESULTATS}

Le Tableau 2 présente les Z-scores et les prévalences de l'émaciation, du retard de croissance et de l'insuffisance pondérale dans la population des enfants malnutris modérés de 6 à 30 mois d'âge. Les résultats indiquent que la consommation de la PFMo a permis une amélioration des indices anthropométriques chez les enfants du groupe d'intervention. En effet, les tests de comparaison des moyennes ont indiqué qu'il n'y avait pas de différence significative entre les valeurs des Z-scores Poids/taille (émaciation), Taille/Age (retard de croissance) et Poids/Age (insuffisance pondérale) des deux groupes d'enfants au début de l'intervention $(\mathrm{P}>0,05)$. A la fin des 6 mois, les Z-scores se sont améliorés de façon significative aussi bien dans le groupe "Etude" que dans le groupe "Témoin" $(\mathrm{P}<0,01)$. Toutefois, ces améliorations étaient beaucoup plus importantes dans le groupe des enfants ayant reçu la PFMo comme complément alimentaire $(\mathrm{P}<0,01)$. Le graphe de la Figure 2 présente l'évolution des prévalences de malnutrition dans les deux groupes. Dans le groupe des enfants ayant reçu la PFMo, aucun enfant ne souffrait plus de malnutrition à la fin des 6 mois (taux de récupération de 100\%).

Dans le groupe "Témoin", après 6 mois, tous les enfants sont sortis de l'état de malnutrition pour ce qui est de l'émaciation et de l'insuffisance pondérale ; mais $2,4 \%$ de 
l'échantillon, présente toujours un retard de croissance modéré. Un risque de rechute est alors à redouter. Dans les cas de l'émaciation et de l'insuffisance pondérale, l'amélioration observée dans la situation nutritionnelle des enfants ne diffère pas significativement par rapport au sexe $(\mathrm{P}>0,05)$ (Tableau 3). En revanche, pour ce qui est du retard de croissance, les Z-scores des enfants de sexe masculin du groupe d'intervention sont statistiquement plus élevés que ceux des enfants de sexe féminin $(\mathrm{P}<0,05)$. Ceci indiquerait une amélioration plus importante de l'état nutritionnel des enfants de sexe masculin.

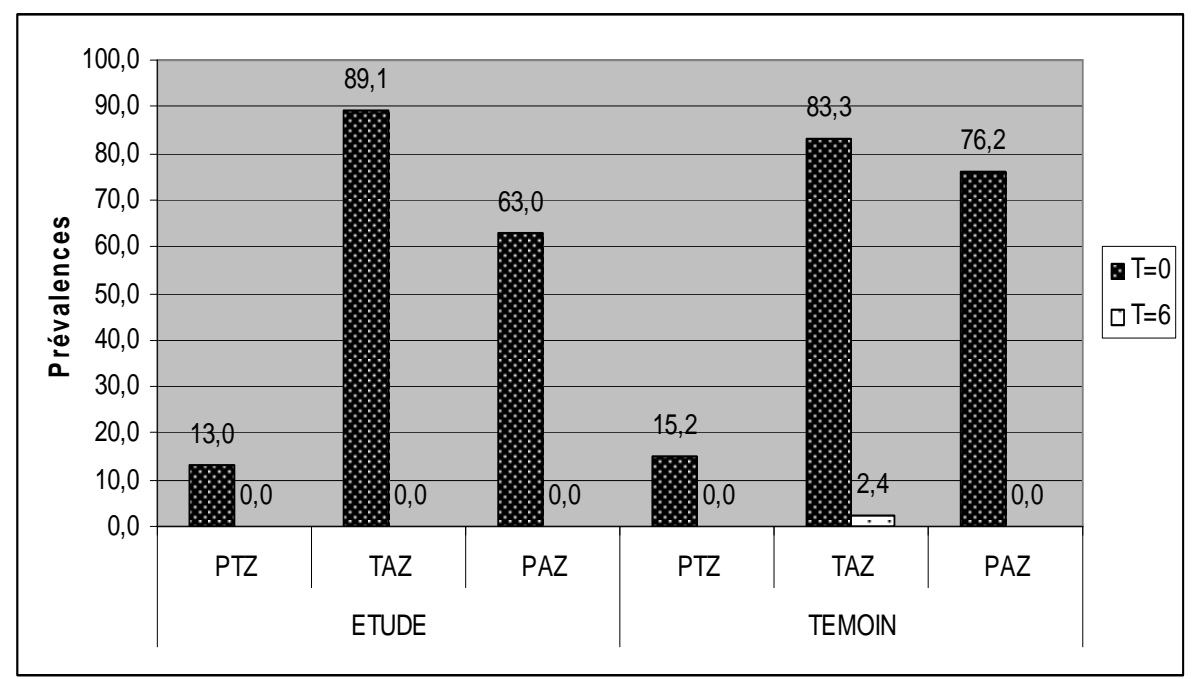

Figure 2 : Evolution des prévalences des différentes formes de malnutrition.

Tableau 1 : Composition de la PFMo et sa contribution à la couverture des besoins d'un enfant de 1 à 3 ans.

\begin{tabular}{|c|c|c|c|c|}
\hline \multirow[t]{2}{*}{ Eléments } & \multirow[t]{2}{*}{ Ff (100g) } & \multirow[t]{2}{*}{ PFMo (100g) } & \multicolumn{2}{|c|}{ TSB d'un enfant de 1 à 3 ans (\%) } \\
\hline & & & $\mathrm{Ff}(100 \mathrm{~g})$ & PFMo $(8 g)$ \\
\hline Protéines (g) & 6,70 & 27,10 & 41,9 & 13,6 \\
\hline Fibres (g) & 0,90 & 19,20 & & \\
\hline Carbohydrates (g) & 13,40 & 38,20 & & \\
\hline Calcium (g) & 440,0 & 2003,0 & 110 & 40 \\
\hline Fer (mg) & 7,00 & 28,20 & 70 & 22,6 \\
\hline Magnésium (mg) & 24,0 & 368,0 & 16 & 20,6 \\
\hline Phosphore (mg) & 24,0 & 204,0 & 2,7 & 2 \\
\hline Vitamine A ( $\mu$ gER $)$ & 6,80 & 18,9 & 443 & 100 \\
\hline Vitamine C (mg) & 120,0 & 17,3 & 1100 & 6,9 \\
\hline
\end{tabular}

Ff : Feuilles fraîches; TSB: Taux de Satisfaction des Besoins ; Source : Lowell (2001) 
Tableau 2 : Prévalences de la malnutrition avant $(\mathrm{T}=0$ mois $)$ et après $(\mathrm{T}=6$ mois $)$ l'intervention (seuil de 5\%).

\begin{tabular}{|c|c|c|c|c|c|}
\hline \multirow[t]{2}{*}{ Groupes } & & \multicolumn{2}{|l|}{ Etude } & \multicolumn{2}{|l|}{ Témoin } \\
\hline & & $\mathbf{T}=\mathbf{0}$ mois & $T=6$ mois & $\mathbf{T}=\mathbf{0}$ mois & $\mathrm{T}=6$ mois \\
\hline \multirow{4}{*}{$\begin{array}{l}\text { Caractéristiques } \\
\text { des échantillons }\end{array}$} & Effectif & 46 & & 42 & \\
\hline & Age (mois) & $15,6 \pm 6,4$ & $21,6 \pm 6,4$ & $16,5 \pm 7,9$ & $22,5 \pm 7,9$ \\
\hline & Poids (kg) & $7,8 \pm 1,2$ & $12,4 \pm 2,3$ & $7,8 \pm 1,2$ & $11,5 \pm 1,9$ \\
\hline & Taille $(\mathrm{cm})$ & $71,1 \pm 6,4$ & $85,5 \pm 7,1$ & $71,8 \pm 7,0$ & $83,6 \pm 7,9$ \\
\hline \multirow{5}{*}{$\begin{array}{l}\text { Emaciation } \\
\text { Poids/Taille }\end{array}$} & Forme sévère $(\%)$ & 2,2 & 0 & 0 & 0 \\
\hline & Forme modérée (\%) & 10,9 & 0 & 15,2 & 0 \\
\hline & Globale (\%) & 13,0 & 0 & 15,2 & 0 \\
\hline & $\mathrm{N}$ & 6 & 0 & 7 & 0 \\
\hline & Z-score & $-1,0 \pm 0,9^{\mathrm{a}}$ & $0,7 \pm 1,0^{\mathrm{b}}$ & $-1,2 \pm 0,9^{\mathrm{a}}$ & $0,3 \pm 0,5^{\mathrm{c}}$ \\
\hline \multirow{5}{*}{$\begin{array}{l}\text { Retard de } \\
\text { croissance } \\
\text { Taille/Age }\end{array}$} & Forme sévère $(\%)$ & 19,5 & 0 & 19,0 & 0 \\
\hline & Forme modérée (\%) & 69,6 & 0 & 64,3 & 2,4 \\
\hline & Globale $(\%)$ & 89,1 & 0 & 83,3 & 2,4 \\
\hline & $\mathrm{N}$ & 41 & 0 & 35 & 1 \\
\hline & Z-score & $-2,6 \pm 0,7^{\mathrm{a}}$ & $0,4 \pm 0,7^{\mathrm{b}}$ & $-2,5 \pm 0,6^{\mathrm{a}}$ & $-0,4 \pm 0,7^{\mathrm{c}}$ \\
\hline \multirow{7}{*}{$\begin{array}{l}\text { Insuffisance } \\
\text { pondérale } \\
\text { Poids/Age }\end{array}$} & Forme sévère $(\%)$ & 6,5 & 0 & 0 & 0 \\
\hline & Forme modérée (\%) & 56,5 & 0 & 76,2 & 0 \\
\hline & Globale $(\%)$ & 63,0 & 0 & 76,2 & 0 \\
\hline & $\mathrm{N}$ & 29 & 0 & 32 & 0 \\
\hline & Z-score & $-2,2 \pm 0,6^{a}$ & $0,7 \pm 0,7^{\mathrm{b}}$ & $-2,3 \pm 0,4^{\mathrm{a}}$ & $0,0 \pm 0,4^{\mathrm{c}}$ \\
\hline & $\mathrm{N}$ & 4 & 0 & 4 & 0 \\
\hline & Z-score & $-0,8 \pm 0,9^{\mathrm{a}}$ & $0,6 \pm 1,0^{\mathrm{b}}$ & $-1,0 \pm 0,9^{\mathrm{a}}$ & $0,4 \pm 0,6^{\mathrm{b}}$ \\
\hline
\end{tabular}

*Les valeurs affectées de la même lettre sur une ligne ne sont pas significativement différentes.

Tableau 3 : Evolution des Z-scores en fonction du sexe des enfants avant $(\mathrm{T}=0$ mois) et après $(\mathrm{T}=$ 6 mois) l'intervention.

\begin{tabular}{|c|c|c|c|c|c|c|c|c|}
\hline \multirow[t]{2}{*}{ Groupes } & \multicolumn{4}{|l|}{ Etude } & \multicolumn{4}{|l|}{ Temoin } \\
\hline & $\mathbf{T}=0$ mois & & $T=6$ mois & & $\mathrm{T}=0 \mathrm{moi}$ & & $T=6 \mathrm{moi}$ & \\
\hline Sexe & Fille & Garçon & Fille & Garçon & Fille & Garçon & Fille & Garçon \\
\hline PTZ & $-1,0 \pm 1,0$ & $-1,0 \pm 0,8$ & $0,7 \pm 1,2$ & $0,7 \pm 0,6$ & $-1,1 \pm 0,9$ & $-1,2 \pm 0,8$ & $0,2 \pm 0,5$ & $0,5 \pm 0,4$ \\
\hline TAZ & $-2,6 \pm 0,7$ & $-2,7 \pm 0,7$ & $0,2 \pm 0,7 *$ & $0,7 \pm 0,7 *$ & $-2,5 \pm 0,6$ & $-2,6 \pm 0,5$ & $-0,5 \pm 0,7$ & $-0,4 \pm 0,7$ \\
\hline PAZ & $-2,2 \pm 0,7$ & $-2,2 \pm 0,5$ & $0,6 \pm 0,9$ & $0,8 \pm 0,5$ & $-2,3 \pm 0,4$ & $-2,3 \pm 0,4$ & $0,0 \pm 0,5$ & $0,2 \pm 0,3$ \\
\hline
\end{tabular}

Les valeurs affectées d'un astérisque(*) sur une ligne sont significativement différentes au seuil de $5 \%$.

\section{DISCUSSION}

Des résultats de cette étude, il ressort que la consommation journalière de $10 \mathrm{~g}$ de PFMo associée à un programme de récupération permet une meilleure amélioration de l'état nutritionnel des enfants présentant une émaciation, un retard de croissance et une insuffisance pondérale modérés. Les avantages nutritionnels liés à la consommation des légumes feuilles en général par les enfants avaient déjà été remarqué dans beaucoup d'études. Ils apportent aux enfants les minéraux, vitamines et facteurs nutritionnels qui manquent souvent dans leur alimentation. Du fait de leur haute valeur nutritive, les légumes feuilles peuvent donc être utilisés pour les enfants en période de croissance (Dansi et al., 2008). Ils permettraient de combler le déficit en nutriments que présentent souvent les aliments habituels lorsqu'ils sont ajoutés à ceux-ci. Guyot et al. (2010) proposaient d'ailleurs la 
supplémentation avec des sources végétales de protéines, lipides et micronutriments tels que les légumes feuilles, comme l'une des démarches pouvant permettre la réduction de la malnutrition. $M$. oleifera est un légume feuilles utilisé en Afrique de l'Ouest et en particulier au Bénin. Il est facile à cultiver, ne nécessite pas un gros investissement pour sa production et est bon marché.

Dans le cas spécifique de la PFMo, les résultats obtenus de la présente étude sont conformes à ceux obtenus par Tété-Bénissan et al. (2012) en utilisant la PFMo comme complément alimentaire pour des enfants malnutris au Togo. Ces auteurs ont remarqué une augmentation du poids et de la taille chez les sujets qui ont consommé régulièrement la PFMo sur une période de plus de 10 semaines seulement. Des résultats similaires ont mêmes été obtenus avec la feuille entière de $M$. oleifera sur des sujets animaux. Ainsi, des bovins recevant cette plante comme supplément gagnaient significativement plus de poids que ceux qui n'en recevaient pas (Nikolaus et al., 2001; Fuglie, 2001). Une expérimentation conduite au Cameroun sur de jeunes cobayes a révélé que les feuilles de $M$. oleifera apparaissaient comme un supplément de choix pour l'amélioration des performances de croissance des cobayes à moindre coût en milieu paysan (Tedonkeng et al., 2005). Ces résultats peuvent être expliqués par le fait que la PFMo étant riche en protéines. En effet, l'enrichissement des plats avec cette poudre améliore les teneurs en protéines (Ndong et al., 2007).

Des résultats de la présente étude, il ressort que la PFMo améliore l'effet du programme de récupération nutritionnelle. Elle semble donc plus indiquée que d'autres suppléments alimentaires tels que la spiruline couteuse et moins efficace (Branger et al., 2002). En effet, la consommation d'une dose journalière de $5 \mathrm{~g}$ de spiruline sur une période de 90 jours par des enfants malnutris au Burkina Faso n'a apporté aucun bénéfice par rapport à la rénutrition traditionnelle (Branger et al., 2002). Toutefois, il faut préciser qu'il existe des différences entre les deux études liées notamment à la période (3 mois pour l'expérience sur la spiruline et 6 mois pour la présente étude). De même, la dose de spiruline utilisée était faible. Une autre étude portant sur une évaluation de l'effet soit de la consommation de PFMo à 3 mois soit de la spiruline à 6 mois dans des conditions similaires permettrait une meilleure comparaison.

D'après Cogill (2003), le retard de croissance n'est pas un bon indicateur pour les interventions ou programmes à court terme (6 à 12 mois). Le retard de croissance indiquant la malnutrition chronique a un caractère cumulatif et est le résultat d'une alimentation déficiente prolongée (Allen et al., 2001). La PFMo présente donc l'avantage d'avoir un effet rapide sur les paramètres de croissance des sujets malnutris en réduisant les niveaux aussi bien de l'émaciation, de l'insuffisance pondérale que du retard de croissance en 6 mois. Elle pourrait donc être conseillée dans des situations qui exigent une récupération rapide. Cependant, il convient de souligner que les mesures anthropométriques manquent de précisions (Maire et al., 2004). Il faudrait alors utiliser d'autres méthodes d'évaluation de l'état nutritionnel pour confirmer les résultats obtenus. Les déterminations du statut en fer et celle du taux d'hémoglobine des enfants semblent à ce sujet important puisqu'il a été rapporté que le fer contenu dans la PFMo est peu biodisponible et par conséquent l'enrichissement des plats avec cette poudre influe peu sur le contenu en fer biodisponible (Ndong et al., 2007). Il a été relevé dans cette étude que les Z-scores pour ce qui est du retard de croissance des enfants de sexe masculin du groupe d'intervention sont statistiquement plus élevés que ceux des enfants de sexe féminin. Les enfants de sexe masculin récupèreraient plus rapidement que leurs homologues féminins du fait de leur propension à manger davantage. Et pourtant, des études antérieures menées dans d'autres localités du Sud-Bénin ont montré que les enfants, quel que soit leur sexe, sont nourris 
de la même manière. Il n'existe alors aucune différence significative dans l'alimentation (aliments consommés, quantités consommées) des filles et des garçons bien que les besoins nutritionnels des enfants de sexe masculin soient plus élevés du fait d'un métabolisme plus élevé (Bodjrènou, 2011). Il serait intéressant que des travaux ultérieurs se penchent sur la question afin que ce phénomène soit élucidé. En effet, les données au plan national ont montré que les prévalences des différentes formes de malnutrition chez les enfants de sexe masculin étaient plus élevées que celles des enfants de sexe féminin. En 2006, cette même tendance avait été observée entre filles et garçons âgés de 6 à 59 mois pour toutes les formes de malnutrition au niveau national (INSAE et Macro Int. Inc., 2007). En 2008, ces différences entre filles et garçons s'étaient révélées significatives dans les cas de l'émaciation et du retard de croissance (AGVSAN, 2009). Toutefois, il faudra tenir compte d'un fait socioculturel vivace qui veut que les enfants de sexe masculin soient vigoureux, donc mieux nourris.

\section{Conclusion}

M. oleifera, une plante bien connue dans les pays tropicaux porte bien empiriquement son nom d'"Arbre miracle" ou d'"Arbre de vie". La PFMo contient un fort potentiel de macronutriments, de micronutriments, des minéraux et des vitamines (Tableau 1). Un enfant de 1 à 3 ans consommant une cuillerée de PFMo, trois fois par jour, couvrirait ses besoins journaliers en protéines $(42 \%)$, calcium $(125 \%)$, magnésium $(61 \%)$, potassium $(541 \%)$, fer $(71 \%)$, vitamine A $(310 \%)$, et vitamine C (22\%). Incorporée à un véhicule alimentaire de toute nature, elle peut être considérée comme un complément alimentaire local et bon marché dans la lutte contre les malnutritions, comme le montre la présente étude. Il ressort des résultats de cette étude que la PFMo a un effet positif sur l'état nutritionnel des enfants âgés de 6 à 30 mois. Une dose journalière de $10 \mathrm{~g}$ de poudre prise comme supplément alimentaire permet de réduire les prévalences et d'améliorer les Zscores des enfants âgés de 6 à 30 mois pour ce qui est de l'émaciation, du retard de croissance et de l'insuffisance pondérale. Une étude sur une durée plus réduite (3 mois par exemple) permettrait d'estimer la période minimale d'efficacité de la poudre. Aussi, l'appréciation de l'état nutritionnel par d'autres méthodes telles que les analyses biologiques confirmerait-elle les résultats de cette étude.

\section{REFERENCES}

Abukutsa-Onyango M. 2007. The diversity of cultivated African leafy vegetables in three communities in western Kenya. African Journal of Food Agriculture Nutrition and Developpement, 7(3). http://www.bioline. org.br/request?nd07021

Afolayan AJ, Jimoh FO. 2009. Nutritional quality of some wild leafy vegetables in South Africa. Int. J. Food. Sci. Nutri., 60(5): 424-431.

AGVSAN (Analyse Globale de la Vulnérabilité, de la Sécurité Alimentaire et de la Nutrition). 2009. Rome, Italie. p 168.

Akubugwo IE, Obasi NA, Chinyere GC, Ugbogu AE. 2007. Nutritional and chemical value of Amaranthus hybridus L. leaves from Afikpo, Nigeria. Afric. $J$. Biotechnology, 6(24): 2833-2839.

Allen LH, Gillespie SR. 2001. What Works? A Review of the Efficacy and Effectiveness of Nutrition Interventions. ACC/SCN nutrition policy paper $n^{\circ} 19$, ADB Nutrition and development series n5. Manila, Philippines.

Amoriggi G. 1980. Technique de Transformation et de Conservation Artisanales des Fruits et Légumes. FAO: Rome (Italie). 1988 ; 62 p.

Avallone S, Brault S, Mouquet $\mathrm{C}$, Trèche $\mathrm{S}$. 2007. Home-processing of the dishes constituting the main sources of micronutrients in the diet of preschool children in rural Burkina Faso. Int. J. Food. Sci. Nutri., 58:108-115. 
Bodjrènou FSU. 2011. Contribution des légumes feuilles à l'alimentation et à la nutrition des enfants de 6 à 35 mois : Cas de la commune de Zè. Thèse d'Ingénieur Agronome, Université d'Abomey-calavi, Bénin. p.149.

Branger B, Cadudal JL, Delobel M, Ouoba H, Yameogo P, Ouedraoga D, Guerin D, Valea A, Zombre C, Ancel P. 2002. La sipiruline comme complément alimentaire dans la malnutrition du nourrisson au Burkina Faso. Koudougou, Burkina Faso. Archives de Pédiatrie, 10(2003) : 424-431.

Cogill B. 2003. Guide de mesure des indicateurs anthropométriques. Projet d'Assistance Technique pour l'Alimentation et la Nutrition, Académie pour le Développement de l'Education, Washington, D.C.

Dansi A, Adjatin A, Vodouhe R, Adeoti K, Adoukonou-Sagbadja H, Falade V, Yedomonhan $\mathrm{H}$, Akoegninou A, Akpagana K. 2008. Biodiversité des légumes-feuilles traditionnels consommés au Bénin. Bibliothèque nationale, Bénin.

FAO. 2009. L'état de l'insécurité alimentaire dans le monde : Crises économiquesrépercussions et enseignements. Rome, Italie.

FAO. 2011. Profil Nutritionnel du BéninDivision de la nutrition et de la protection des consommateurs. http://faostat.fao.org/ site/666/default.aspx (consulté en Novembre 2012).

Fuglie LJ. 2011. The natural nutrition for the tropics. In The miracle tree, the multiple attributes of Moringa, Fuglie LJ (ed). CTA, CWS : Dakar, Sénégal ; 103-115.

Guyot J-P, Mouquet-Rivier C, Verniere C. 2010. La nutrition, un défi pour la planète : Amélioration de la qualité nutritionnelle des aliments. IRD, SUDS : Les dossiers thématiques de l'IRD.

Houinsou E. 1998. Identification approfondie des Organisations Professionnelles de la Filière Fruits et Légumes Séchés au Bénin. Rapport définitif. Programme «Entreprenariat au Bénin ».
INSAE et Macro International Inc. 2007. Enquête Démographique et de Santé Bénin (EDSB-III). Institut National de la Statistique et de l'Analyse Economique (INSAE), Bénin et Macro International Inc. Calverton, Maryland, USA. (disponible à http://www.measuredhs. com/pubs/pub_details.cfm?ID=733\&ctry_i $\mathrm{d}=52 \& \operatorname{SrchT} \mathrm{p}=$ ctry \&flag=sur \&cn=Benin).

Maire B, Delpeuch F. 2004. Indicateurs de Nutrition pour le Développement : Guide de Référence. FAO: Rome, Italie.

Ndong MWS, Dossou N, Guiro AT, Gning RD. 2007. Valeur nutritionnelle du Moringa oleifera, étude de la biodisponibilité du fer, effet de l'enrichissement de divers plats traditionnels sénégalais avec la poudre des feuilles. African Journal of Food Agriculture Nutrition and Developpement, 7 (3). http://www.bioline.org.br

Nikolaus F, Makkar HPS, Becker K. 2001. The Potential of Moringa oleifera for Agricultural and Industrial Uses. The Miracle Tree, the Multiple Attributes of Moringa, Fuglie LJ (ed). CTA, CWS: Dakar, Sénégal; 45-76.

Schippers RR. 2002. African indigenous vegetables an overview of the cultivated species. Chatham, UK. Natural Resources Institute/ACP-EU Technical Centre for Agricultural and rural Cooperation. In ABUKUTSA-ONYANGO M. 2007. The diversity of cultivated african leafy vegetables in three communities in western Kenya. African Journal of Food Agriculture Nutrition and Developpement, 7(3): 15.

Tchiegang C, Kitikil A. 2004. Données ethnonutritionnelles et caractéristiques physico-chimiques des légumes-feuilles consommés dans la savane de l'Adamaoua (Cameroun). Tropicultura, 22: 11-18.

Pamo ET, Niba AT, Fonteh FA, Tedonkeng F, Kana JR, Boukila B, Tsachoung J. 2005. Effet de la supplémentation au Moringa oleifera ou aux blocs multinutritionnels sur l'évolution du poids post partum et la 
croissance pré-sevrage des cobayes (Cavia porcellus L.). Livestock Research for Rural Development 17(4): 9 pp; available from: http://www.lrrd.org/lrrd17/4/ tedo17046.htm

Tété-Bénissan A, Lawson-Evi KA, Kokou K, Agbeassor M. 2012. Effet de la consommation de la poudre de feuilles de Moringa oleifera Lam. sur l'évolution du profil de l'hémogramme des enfants malnutris au Togo: évaluation chez les sujets HIV positifs. African Journal of Food Agriculture Nutrition and Developpement, 12(2). www.bioline.org. $\mathrm{br} / \mathrm{abstract}$ id=nd12023\&lang=fr
Unicef. 2010. Overview of children in west and central Africa. Site officiel visité le 27/10/2010.

Yang RY, Chang LC, Hsu JC, Weng BBC, Palada MC, Chadha ML, Levasseur V. 2007. Nutritional and functional properties of moringa leaves - from germplasm to plant, to food, to health. In Moringa Leaves: Strategies, Standards and Markets for a better impact on nutrition in Africa. Moringanews, Moringa and Plant Resources Network: Paris; 9 pp. 